\title{
Virus induced gene silencing (VIGS) for functional analysis of wheat TaPCNA participated in apoptosis exposed to enhanced UV-B radiation
}

\section{Meiting Du}

Shanxi Normal University

\section{Ying Zhang}

Shanxi Normal University

Haiyan Ran

Shanxi Normal University

Rong Han

Shanxi Normal University

Huize Chen ( $\nabla$ chenhuize@hotmail.com )

Shanxi Normal University https://orcid.org/0000-0003-2161-0089

\section{Research}

Keywords: VIGS, UV-B radiation, PCNA, Apoptosis

Posted Date: December 23rd, 2019

DOI: https://doi.org/10.21203/rs.2.19488/v1

License: (c) (i) This work is licensed under a Creative Commons Attribution 4.0 International License. Read Full License 


\section{Abstract}

Background:Plant cell apoptosis is manifested by breakage of cellular DNA and changes of membrane permeability which caused physiological metabolism disturbance and growth inhibition. Besides, the UV$B$ radiation inhibits plant growth and DNA break. The PCNA (Proliferating Cell Nuclear Antigen) is an important factor participating in cell proliferation and DNA repair. Here, we explored the function of TaPCNA in wheat exposed to enhanced UV-B radiation by means of BSMV-VIGS.

Results:The results showed the expression of TaPCNA was down-regulated by VIGS. Wheat leaves presented several spots after virus inoculation, and they were also found significantly curly and the whole plants were shorter than that of the control. The growth rate of wheat in each treatment was significantly inhibited after gene silencing (BSMV::TaPCNA). Especially, the growth of the gene silencing group was almost stagnant after processed with enhanced UV-B radiation (BSMV::TaPCNA+B). Also, the growth of uninoculated plants was suppressed after being exposed to enhanced UV-B radiation (WT+B). The cell length changed obviously, and the BSMV::TaPCNA group showed to be smaller cell than those of WT and BSMV:00 group. The physiochemical and metabolisms parameters were determined in different groups. The contents of soluble sugar and soluble protein decreased while the content of MDA increased in BSMV::TaPCNA group. In addition, the SOD activity enhanced while that of POD and CAT declined. These results indicated that the antioxidant system was destroyed after TaPCNA was silenced by VIGS. After exposed to enhanced UV-B radiation, the wheat growth in different group was less notable than TaPCNA silencing. Either in the BSMV:TaPCNA-1 or BSMV:TaPCNA-2 group. Both BSMV::TaPCNA group and BSMV::TaPCNA+B group demonstrated accumulation of small molecular weight DNA fragments and micronucleus through the DNA laddering and the PI staining.

Conclusions: In summary, our results preliminarily illustrated the function of TaPCNA in apoptosis exposed to enhanced UV-B radiation and thus provided an idea for researchers to explore the function of certain genes in such a complex wheat genomes and offered theoretical basis to improve wheat agronomic trait.

\section{Background}

Proliferating Cell Nuclear Antigen (PCNA) is an evolutionally conserved protein found in all eukaryotic species and archaeas. PCNA was initially identified as a co-factor for DNA polymerase $\delta$, needed for DNA synthesis during its replication[1-3]. More than 30 years ago, PCNA was identified as an antigen of autoimmune disease in the serum of patients with systemic lupus erythematosus. Subsequent studies showed the expression level of PCNA is associated with proliferation or tumor transformation[5-6]. In addition to DNA replication, PCNA also functioned in many other important cellular processes, such as chromatin assembly, DNA damage repair, sister chromatid aggregation, and cell cycle regulation[7]. Further experiments indicated that PCNA has the same function as that of cyclin[8]. 
During the life cycle of a plant, cells would experince a certain number of divisions. As plants are continuously exposed to harmful factors (endogenous and environmental), genetic material could be damaged in many ways. The accumulation of spontaneous or induced mutations in many divised cells may disrupt the normal functioning of the whole plant. The biological effects of mutagens depend on the type of DNA damage they caused that led to uncontrolled cell proliferation or death. There are many chemical or physical factors resulting in DNA damage. Endogenous mutagens mainly include reactive free radicals, such as hydroxyl radicals, nitric oxide, superoxide anions, while environmental mutagens mainly include environmental mutagens, from ultraviolet radiation, ionizing radiation, to chemical substances. The stable inheritance of undamaged genetic information requires the development of different DNA repair systems, such as Base Excision Repair (BER), Nucleotide Excision Repair (NER), Mismatch Repair (MR), and Double-Strand Break Repair (DSBR). The knowledge of eukaryotic DNA repair systems came primarily from the study of yeast and animals[8].

Wheat is one of the earliest cultivated and widely distributed crops providing basic food for people all over the world[9]. Lights are needed for wheat for photosynthesis. While UV-B radiation is a component of sunlight and a critical factor on wheat growth. However, the contents of ozone layer reduced due to the presence of large amounts of chlorofluorocarbons (CFCS) around the earth which has cut dowm the capability of ozone layer to absorb UV-B radiation in recent years. The UV-B radiation on the earth surface will increase by $20-40 \%$ from 2010 to 2020 as estimated by the GISS model[10]. In addition, people find the ozone hole appears in the earth's the North Pole for the first time[11], indicating a terrible situation of the destroyed ozone layer. UV-B radiation affects a variety of organisms in the ecosystem, crops in particular[12-15]. Wheat is one of the most important food crops for human beings, and it is of great significance to study the stress characteristics of wheat to UV-B radiation[16].

From the above, PCNA is involved in the process of DNA replication and cell division. It is necessary to carry out researcher on how PCNA affects the normal physiological metabolism of wheat after UV-B radiation. We generated BSMV::TaPCNA vectors for target silencing of wheat leaf genes using BSMV VIGS. The results showed TaPCNA is crucial in DNA damage and apoptosis when wheats are exposed to UV-B radiation. This study will provide some new insights into the subsequent exploration of plant and environmental factors.

\section{Materials And Methods}

\section{Plant material and growth conditions}

Yimai325 (Triticum aestivum L. cv.) was used as research materials. Plants were growth in the illumination incubator with temperature $22-25^{\circ} \mathrm{C}$, with a humidity of $60 \%$, light cycle $8 / 16 \mathrm{~h}$ (8 hours light and 16 hours dark). N. benthamiana were grown in $20-25^{\circ} \mathrm{C}$, light cycle was $14 / 10 \mathrm{~h}$ (14 hours light and 10 hours dark). The UV-B radiation dose was $10.08 \mathrm{KJ} \cdot \mathrm{m}^{-2} \cdot \mathrm{d}^{-1}$, and irradiation time was $8 \mathrm{~h}$. The gene silenced plants were irradiated under the white light or combined with UV-B radation for 7 days after virus infection. 


\section{Construction of BSMV VIGS vectors}

The bioinformatics analysis of PCNA was carried out by EMBL-EBI and NCBI web site. LIC (Ligation Independent Clone) was used to carry on vector attachment, and LIC cloning site was added on the specific primer for gene RT-PCR (GenBank accession: KM087781.1). By referring to the research of Cheng Yuan et al.[17], the specific linking mode and LIC specific cloning site were obtained. The products were transformed into E.coli DH5a after the completion of the ligation. The positive colone were screened according to the antibiotics resistance, and colony PCR were also used for the identification.

Agroinfiltration of $\mathrm{N}$. benthamiana and viral inoculation on Wheat

The pCaBS- $a, p C a B S-\beta$ and $p C a-\gamma$ were transformed into Agrobacterium GV3101 respectively. Colonies were picked into $10 \mathrm{ml}$ YEB liquid medium with antibiotics, shaked $150 \mathrm{rpm}$ at $28{ }^{\circ} \mathrm{C}$ for $10-12 \mathrm{~h}$. The bacterias were collected by $4,000 \mathrm{rpm}$ centrifugation at $\mathrm{OD}_{600}$ is 1.8. Resuspended the Agrobacterium bacteria with suspension buffer and made the $\mathrm{OD}_{600}=0.7$ or so. Then, the mixture will be used to inject into the four to eight in the $\mathrm{N}$. benthamiana leaf stage. After inoculation for 7-12 days, tobacco leaves were grinded into the wheat inoculation buffer for the infection on wheat leaves. After wearing surgical gloves, the forefinger and thumb were dipped into the inoculation buffer and rub the wheat leaves which were sprayed with emery.

\section{RNA extraction and semi-quantitative RT-PCR analysis}

The total RNA was extracted by Trizol method, the materials of each treatment group were strictly quantified at the initial stage of extraction, and the concentration was determined and unified for the synthesis of the first strand of CDNA after extraction. Then RT-PCR was performed and the results were subjected to agarose gel electrophoresis and the gray scale values were analyzed.

\section{Determination of physiochemical and metabolisms}

MDA content was measured by thiobarbituric acid (TBA) reaction. The SOD activity was assayed by measuring its ability to inhibit the photochemical reduction of NBT. The POD activity was determined by monitoring the formation of guaiacol dehydrogenation product via following the rise of absorbance at $470 \mathrm{~nm}$ over $1 \mathrm{~min}$. The CAT activity was determined by monitoring the decomposition of $\mathrm{H}_{2} \mathrm{O}_{2}$ at 240 $\mathrm{nm}[18]$. Determination of Soluble sugar and Soluble protein content were performed by means of anthrone method and coomassie blue staining, respectively.

\section{DNA extraction and detection}

The modified CTAB method was improved according to Amani abdel-latif et al[19]. There was $0.1 \mathrm{~g}$ materials takenfor grinding, the precooling - CTAB Buffer $(50 \mathrm{mM}$ Tris - $\mathrm{Hcl} \mathrm{pH}=8.0,0.7 \mathrm{M} \mathrm{NaCl}, 10 \mathrm{mM}$ EDTA $-\mathrm{Na}_{2} \mathrm{pH}=8.0$ ) and $450 \mu \mathrm{L} 65^{\circ} \mathrm{C}$ CTAB Buffer (-CTAB Buffer adding 20\% CTAB and $40 \mathrm{mM} \beta$ mercaptoethanol) added, $65^{\circ} \mathrm{C}$ water bath heat preservation for $1 \mathrm{~h}$, centrifugal 10,000 for rpm 2 min, after taking supernatant, $600 \mu \mathrm{L}$ chloroform/isoamyl alcohol (24:1) was added, then went reverse 
blending, centrifugal, upper liquid to $1.5 \mathrm{~mL}$ centrifuge tube, add volume isopropyl alcohol, such as precipitation $-20{ }^{\circ} \mathrm{C}$ for 30 min, centrifugal, abandon supernatant, precooling precipitation with $76 \%$ ethanol and $70 \%$ ethanol for cleaning, abandoned after centrifugal net ethanol, add pure water dissolve DNA after dried, then preserved at $-20^{\circ} \mathrm{C}$. Agarose gel was used to detect the extracted DNA, and observing DNA laddering phenomenon.

\section{PI staining}

The leaves were cut into squares by $0.5 \times 0.5 \mathrm{~cm}^{2}$, and PI solution with a concentration of $1 \mu \mathrm{g} / \mathrm{mL}$ was prepared. The leaves were vacuumized in a $1 \mathrm{~mL}$ glass syringe for about $30 \mathrm{~s}$, rinsed with water and observed with fluorescence microscope.

\section{Data analysis}

LSD and Duncan multiple range test were used for significance difference analysis by SPSS.

\section{Results}

\section{Bioinformatics analysis of TaPCNA}

The structure domain of PCNA was conservative, and the topological structure of N-terminal and Cterminal was similar upon analysis of the protein structure via online website. Three PCNA molecules formed a loop closed to DNA double helix. In the presence of limitation on carried gene fragments of the virus vector, the PCNA was divided into two fragments based on both ends of $\mathrm{N}$-terminal and $\mathrm{C}$-terminal in this research, which were respectively named PCNA-1 (400bp) and PCNA-2 (402bp).

\section{Agrobacterium mediated BSMV VIGS in N.benthamiana and Wheat}

Figure 2a showed the 7-15 days N.benthamiana leaves after inoculated with BSMV VIGS mediated Agrobacterium. Among the three treatments inoculated with virus vectors, BSMV:00 showed virus symptoms. BSMV:TaPCNA-1 and BSMV:TaPCNA-2 showed different degrees of leaf edge curling, small leaf area and plant dwarfing. Considering the evolutionary conservatism of PCNA, it is similar to the genesilencing phenotype reported by Gareth Bruce et al.[20], indicating that N.benthamiana was successfully infected with the virus. We inferred that the phenotype changed since the PCNA is a key factor on meristem growth. After BSMV::TaPCNA infected the wheat, the overall growth of the plant was weak and dwindled that observed at 13-15 days (Fig. $2 b$ and 2 c). Also, wheat leaves showed some signs of virus infection.

The growth rate of the gene silenced plants was statistically calculated (Fig. 3). The 5-day old wheat seedlings were inoculated, and the calculation began on the $10^{\text {th }}$ day and lasted until the $27^{\text {th }}$ day. $B S M V: T a P C N A-1$ and BSMV:TaPCNA-2 groups showed obvious growth stagnation on the 15th day during the growth period (at 10 days after inoculation). While the growth rates of WT and BSMV:00 was slow during plant growth to the later stage. And the growth rate dropped slowly with the extension of the 
growth cycle. In order to prove the plants growth stagnation is a result of gene silencing, the length of mesophyll cells was recorded and caculated (Fig. 4). It was found that the cell length of BSMV:TaPCNA-1 and BSMV:TaPCNA-2 groups were significantly shorter than WT and BSMV:00. And the WT and BSMV:00 was not significantly different.

\section{The expression of TaPCNA after VIGS and exposed to enhanced UV-B radiation}

The phenotype of wheat processed with VIGS maintained for 15-25 days. However, the plants can recover at the $30^{\text {th }}$ day after inoculations. Therefore, total RNA was extracted from 22 days wheat plants after gene silencing , and semi-quantitative RT-PCR was performed to determine the efficiency of VIGS. The expression of TaPCNA in BSMV:TaPCNA-1 and BSMV:TaPCNA-2 groups decreased significantly (Fig. 5), indicating that the BSMV::TaPCNA interfered the expression of TaPCNA. To test the response of TaPCNA to enhanced UV-B radiation, we extracted total RNA from seedlings after irradiated with enhanced UV-B radiation. The RT-PCR showed the expression level increased in leaves, whilethe expression reduced in root (Fig. 5). We can infer that the reduced expression of TaPCNA in root is a result of compenstroy function under enhanced UV-B radiation. Thus the germination could get more energy and restore the normal growth.

\section{Changement of physiochemical and metabolisms of Wheat after VIGS and treated with enhanced UV-B radiation}

Peroxidase (POD) was associated with respiration, photosynthesis and auxin oxidation of plants. The activity of POD was measured on the 7th day after TaPCNA silencing (Fig. 6). The results showed the activity of POD in BSM::00 group was not significantly different from WT, while the activity of POD in $B S M V:: T a P C N A-1$ and BSMV::TaPCNA-2 were decreased obviously compared with WT and BSMV::00. The activity of POD on the $7^{\text {th }}$ day was much higher than that on the $1^{\text {st }}$ day after virus infection. These results indicated that silenced TaPCNA affected plant growth and caused tremendous accumulation of peroxides affecting the overall growth of the plant. Catalase (CAT) can remove the excess accumulated $\mathrm{H}_{2} \mathrm{O}_{2}$ and indirectly reflect the stress resistance and metabolic intensity of plants (Fig. 6). The activity of CAT in BSMV::TaPCNA-1 and BSMV::TaPCNA-2 was lower when compared with WT and BSMV::00. The concentration of Malondialdehyde (MDA) is a reflection of the degree of lipid peroxidation in plant cell membrane (Fig. 6). The MDA content in BSMV::TaPCNA-1 and BSMV::TaPCNA-2 content increased when compared with WT and BSMV::00. Besides, MDA was proven a substance product of membrane lipid under the action of reactive oxygen species (ROS), indicating that the membrane was damaged after the silencing of TaPCNA. Superoxides dismutase (SOD) was functioned as remove free oxygen radicals in cells. As shown in figure 6, the activity of SOD of BSMV::TaPCNA-1 and BSMV::TaPCNA-2 were decreased compared with WT and BSMV::00. After treated with enhanced UV-B radation, the activity of POD and CAT were decreased more and SOD activity and MDA content increased more in each treatment group. Moreover, it was found WT+B and BSMV::TaPCNA-1+B and BSMV::TaPCNA-2+B showed the same trend. Soluble sugar and soluble protein are useful parameters on reflect the metabolic process of plants. As shown in figure 6, the content of soluble sugar and soluble protein in BSMV::TaPCNA-1 and 
$B S M V:: T a P C N A-2$ decreased a lot compared with WT and BSMV::00. These indicated that the metabolism of plants were slowed down. It also indirectly proves that silence of TaPCNA affected plant growth. Especially, after being exposed to enhanced UV-B radation the reduction was exacerbated.

\section{Apoptosis in wheat leaves after VIGS and exposed to enhanced UV-B radiation}

The DNA of wheat plants in TaPCNA silencing was extracted. As shown in figure 7, the gel electrophoresis showed the complete DNA bands of WT and BSMV::00, while the DNA of BSMV::TaPCNA-1 and $B S M V: T a P C N A-2$ were dispersed as the DNA laddering. Both $\mathrm{WT}+\mathrm{B}$ and $B S M V:: 00+\mathrm{B}$ groups showed regular low-molecular weight fragments, and similar fragmentation and dispersion with $B S M V:: T a P C N A-$ 1+B and BSMV::TaPCNA-2+B, presenting that the DNA damage and apoptosis in wheat cells had happend.

To confirm the apoptosis in wheat cells after TaPCNA silenced, the PI staining was carried out on the VIGS treated leaves. According to figure 8, the dead cells showed obvious contour. The view of WT and $B S M V:: 00$ was dark heavy while BSMV::TaPCNA-1 and BSMV::TaPCNA-2 showed more shiny colored parts than WT and $B S M V:: 00$. After magnified, normal cells showed clear notch red. The apoptosis cells nucleus could be stained in red. This indicated the inhibition of wheat growth caused by TaPCNA silencing induced apoptosis. PI staining was also carried out for each treatment group after UV-B radiation. As shown in figure 8 , after treated with enhanced UV-B radiation all groups could be observed and with different degrees of red colored parts. While BSMV::TaPCNA-1+B and BSMV::TaPCNA-2+B showed more colored parts than $W T+B$ and $B S M V: 00+B$.

\section{Discussion}

The TaPCNA was presented with important functions consistent with previous studies, especially in the growth of meristem tissue, DNA damage repair and other process in the whole lifecycle [2]. Here, the intact amino acids sequence of TaPCNA protein were divided into two fragments, as TaPCNA-1 and TaPCNA-2. The results indicated these two fragments showed same function although they are different in the end of the protein. Previous studies reported the structure of PCNA is made up of three molecules to form closed loop structure which both ends of topology structure is similar, which could be the reason that the protein structure made TaPCNA-1 and TaPCNA-2 showed no difference in function. In the future, we will explore the difference between the $\mathrm{N}$-terminal and $\mathrm{C}$-terminal of this protein in detail at protein level.

Virus induced gene silencing technology is a useful and reliable tool to investigate gene functions study and disease research in many crop, since the absence of key genes plants can show obvious phenotype [21]. While, in some other aspets on genes functions study, the VIGS could not be the best way, for this technology can be used in some transgenic plants not all the life cycle [22]. In the later experiments, we can explore some convenient inoculation techniques that can be carried out in the early stage of plants to expand the use period of this technology and make it a larger scale when using. At last, we can also 
explore more gene editing techniques in wheat to study genes function and provide more theoretical basis to improve wheat agronomic trait.

\section{Conclusion}

As the importance of TaPCNA in the plant life cycle, our findings showed TaPCNA is crucial in DNA damage and apoptosis when wheats are exposed to UV-B radiation. This study will provide some new insights into the subsequent exploration of plant and environmental factors.

\section{Declarations}

\section{Acknowledgement}

We are grateful to Quanshijin Company for excellent technical support. We thank Hailong Tie for helping us plant material.

\section{Availability of data and materials}

Please contact the corresponding author for data requests.

\section{Funding}

This work was supported by the Natural Science Foundation of China under Grant No. 31900251.

\section{Ethics approval and consent to participate}

Not applicable.

\section{Consent for publication}

Not applicable.

\section{Competing interests}

The authors declare that they have no competing interests.

\section{Author's contributions}

Meiting and Huize proposed the idea and designed the experiments. Both Ying and HaiYan drafted the manuscript, while Rong helped analyse the results. All authors read and approved the final manuscript.

\section{References}

1. Tan $C K$, Castillo $C S$, So $A G$, et al. An auxiliary protein for DNA polymerase $\Delta$ from fetal calf thymus. Journal of Biological Chemistry, 1986, 261(26):12310-12316. 
2. Bravo R , Frank R, Blundell P A, et al. Cyclin/PCNA is the auxiliary protein of DNA polymerase. Nature, 1987, 326(6112):515-517.

3. Prelich G, Tan C K, Kostura M , et al. Functional identity of proliferating cell nuclear antigen and a DNA polymerase- $\delta$ auxiliary protein. Nature, 1987, 326(6112):517-520.

4. Miyachi K. Auroantibody to a nuclear antigen in proliferating cells. J Immunol, 1978, 121.

5. Bravo R, Fey S J, Bellatin J, et al. Identification of a nuclear polypeptide ("cyclin") whose relative proportion is sensitive to changes in the rate of cell proliferation and to transformation. Progress in Clinical \& Biological Research, 1982, 85 Pt A:235.

6. Bravo R .Cyclin: a nuclear protein whose level correlates directly with the proliferative state of normal as well as transformed cells. Leukemia Research, 1984, 8(2):143-157.

7. Maga G , Hubscher U . Proliferating cell nuclear antigen (PCNA): a dancer with many partners. Journal of Cell Science, 2003, 116(15):3051-3060.

8. Strzalka W, Ziemienowicz A. Proliferating cell nuclear antigen (PCNA): a key factor in DNA replication and cell cycle regulation. Annals of Botany, 2011, 107(7):1127-1140.

9. Heisey P W, Lantican M A, Dubin H J. Impacts of international wheat breeding research in developing countries, 1966-97. CIMMYT, 2002.

10. Taalas P , Amanatidis G T, Heikkil A . European Conference on Atmospheric UV Radiation: Overview. Journal of Geophysical Research, 2000, 105(D4):4777.

11. Manney G L, Santee M L , Rex M , et al. Unprecedented Arctic ozone loss in 2011. Nature. 478(7370): 469.

12. Lv Z W , Zhang X S , Liu L K, et al. Comparing intraspecific responses of 12 winter wheat cultivars to different doses of Ultraviolet-B radiation. Journal of Photochemistry and Photobiology B: Biology, 2013, 119:1-8.

13. Zhao S , Huang Q, Yang P , et al. Effects of Ion Beams Pretreatment on Damage of UV-B Radiation on Seedlings of Winter Wheat (Triticum aestivum). Applied Biochemistry \& Biotechnology, 2012, 168(8):2123-2135.

14. Yang L, Han R, Sun Y. Damage repair effect of He-Ne laser on wheat exposed to enhanced Ultraviolet-B radiation. Plant Physiology \& Biochemistry, 2012, 57(8):218-221.

15. É. C. Schmidt, Nunes B G, Maraschin M, et al. Effect of ultraviolet-B radiation on growth, photosynthetic pigments, and cell biology of Kappaphycus alvarezii (Rhodophyta, Gigartinales) macroalgae brown strain. Photosynthetica, 2010, 48(2):161-172.

16. Chen $\mathrm{H}$, Han R . F-actin participates in the process of the "partition-bundle division". Russian Journal of Plant Physiology, 2015, 62(2):187-194.

17. Yuan C, Li C , Yan L, et al. A High Throughput Barley Stripe Mosaic Virus Vector for Virus Induced Gene Silencing in Monocots and Dicots. PLOS ONE, 2011, 6(10):e26468.

18. Liu W , Xu F , Lv T, et al. Spatial responses of antioxidative system to aluminum stress in roots of wheat (Triticum aestivum, L.) plants. Science of The Total Environment, 2018, 627:462-469. 
19. Abdel-Latif A , Osman G . Comparison of three genomic DNA extraction methods to obtain high DNA quality from maize. Plant Methods, 2017, 13(1):1.

20. Bruce $G, G u M$, Shi $N$, et al. Influence of retinoblastoma-related gene silencing on the initiation of DNA replication by African cassava mosaic virus Rep in cells of mature leaves in Nicotiana benthamianaVirology Journal, 2011, 8(1):1-6.

21. Lee WS, Rudd JJ, Kanyuka K: Virus induced gene silencing (VIGS) for functional analysis of wheat genes involved in Zymoseptoria tritici susceptibility and resistance. Fungal genetics and biology : FG \& B 2015, 79:84-88.

22. Bennypaul HS, Mutti JS, Rustgi S, Kumar N, Okubara PA, Gill KS: Virus-induced gene silencing (VIGS) of genes expressed in root, leaf, and meiotic tissues of wheat. Funct Integr Genomics 2012, 12(1):143-156.

\section{Figures}

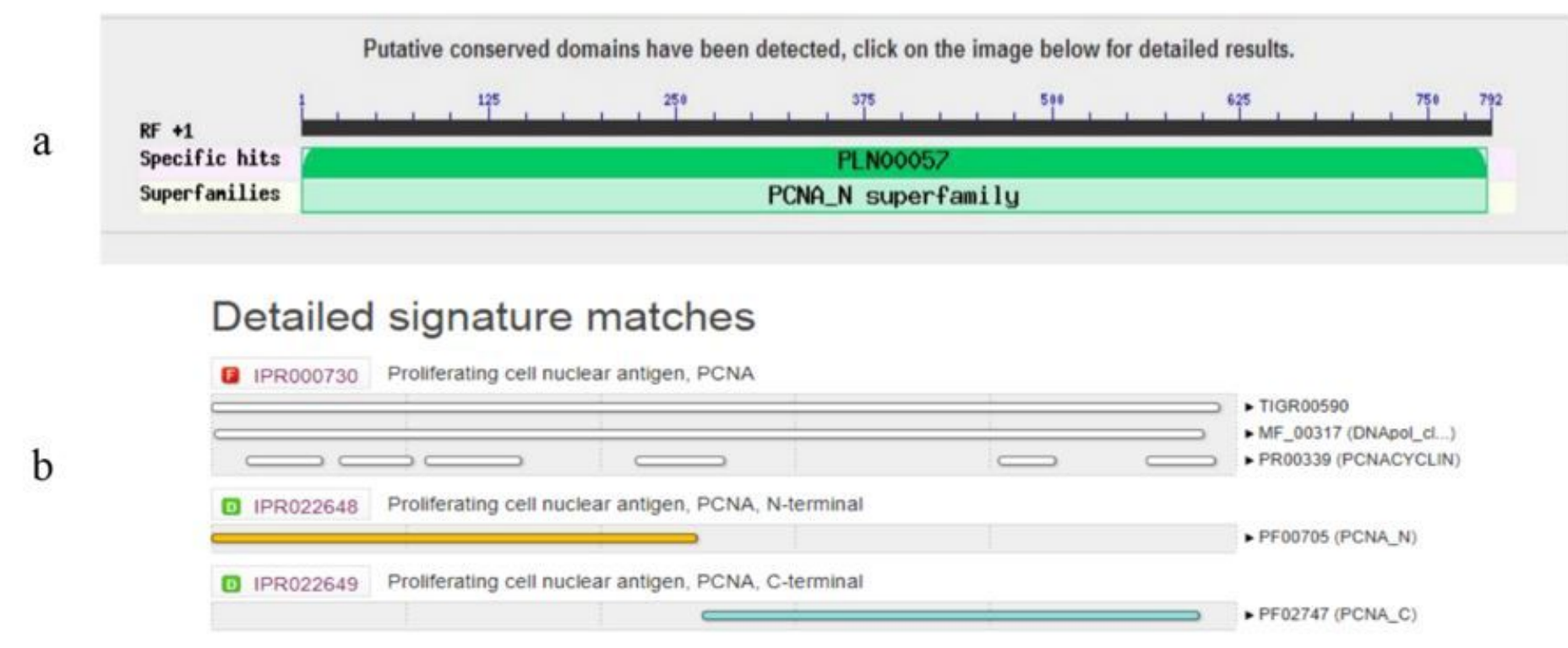

\section{Figure 1}

PCNA protein structure analysis. (a) PCNA domain from NCBI blast. PCNA belongs to the PCNA_N superfamily. (b) PCNA domain structure from EMBL-EBI blast. The results showed N-terminal of PCNA is 1-125 amino acids while the $\mathrm{C}$-terminal is 127-254 amino acids. 
a

WT

BSMV:00

BSMV:Tapcna-1

BSMV:Tapcna-2

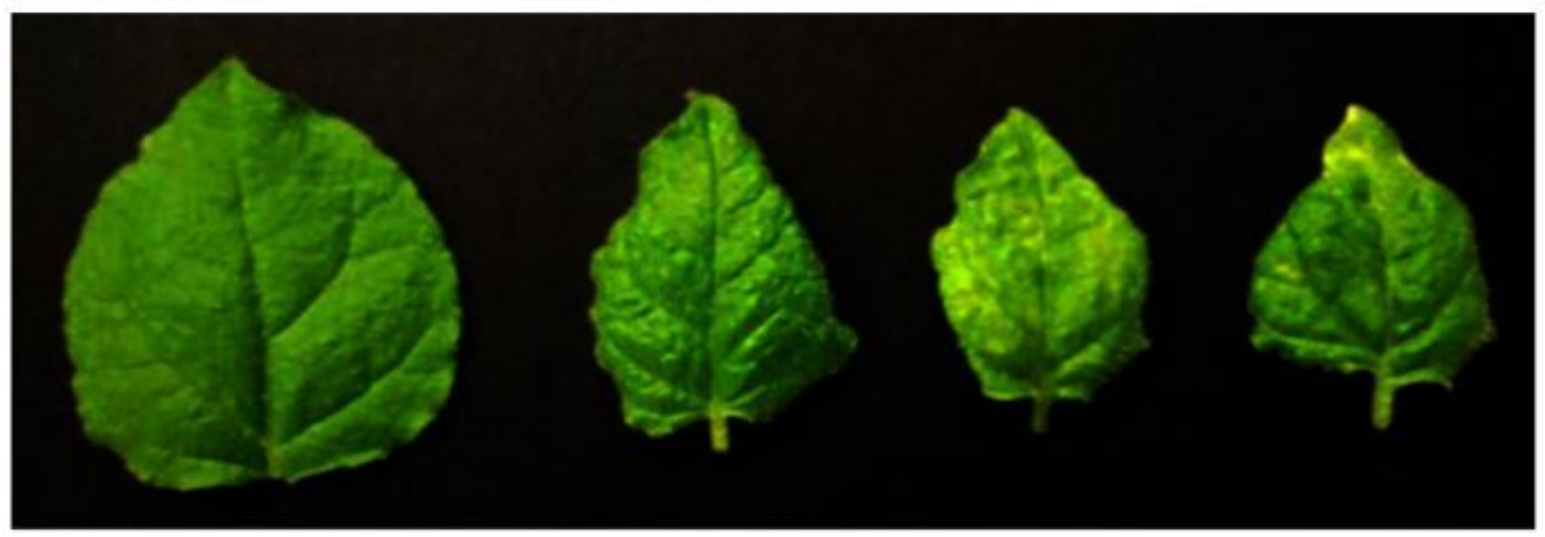

b

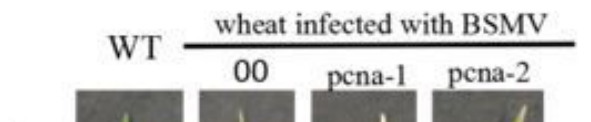

WT

BSMV:00

BSMV:Tapcna-1 BSMV:Tapcna-2

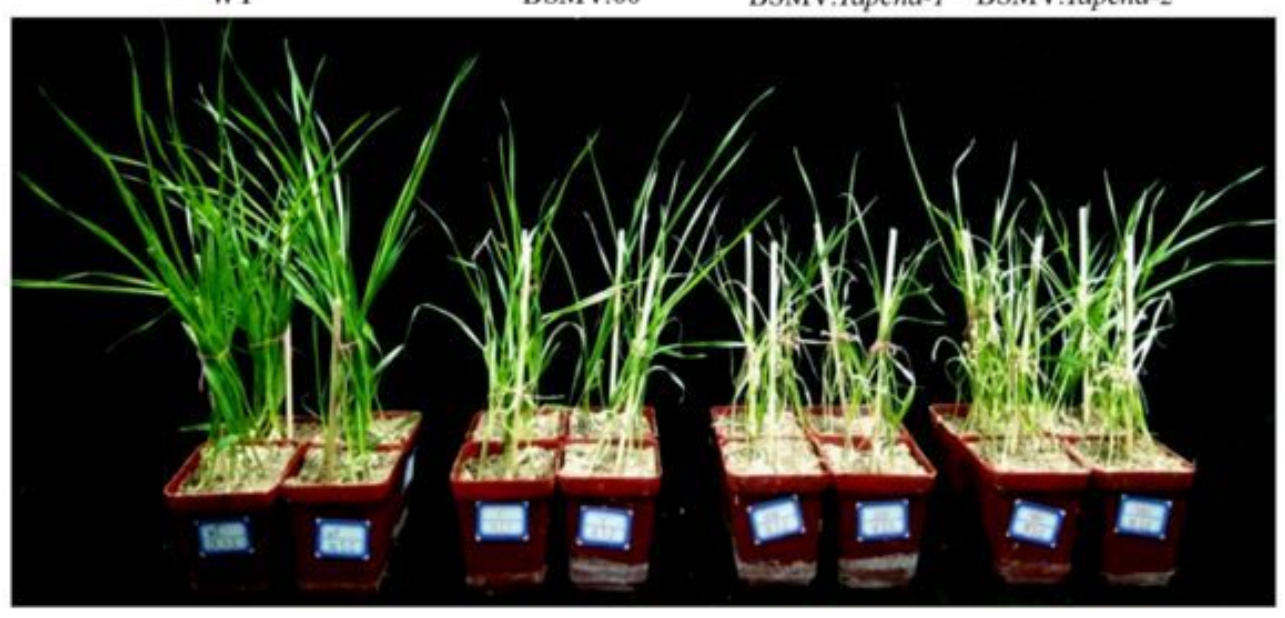

C
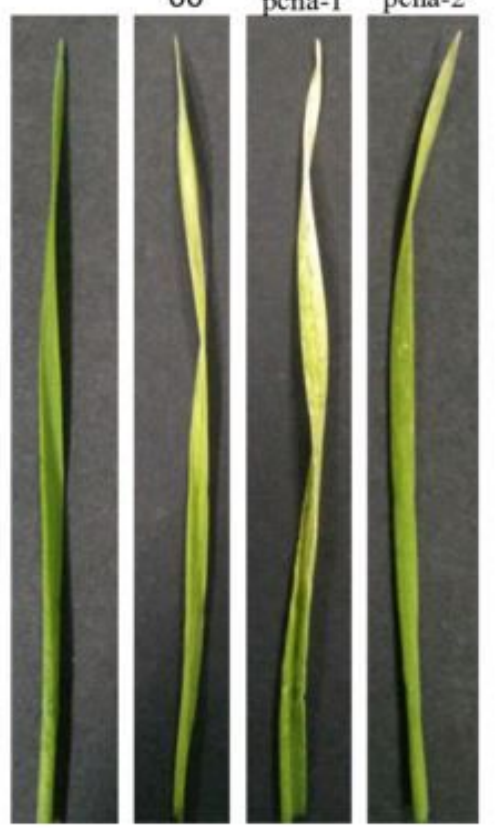

\section{Figure 2}

Infection after virus inoculated into N.benthamiana and wheat leaves. (a) The phenotype of N.benthamiana leaves showed abnormal leaf development and growth retardation. (b) Wheat was presented as dwarf after PCNA gene silencing. (c) The arrow showed changes of virus infection. 
WT

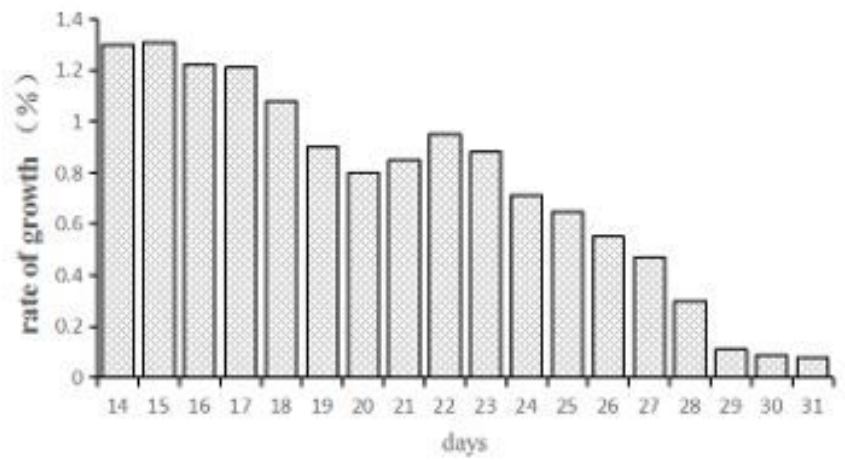

BSMV:Tapcna-1

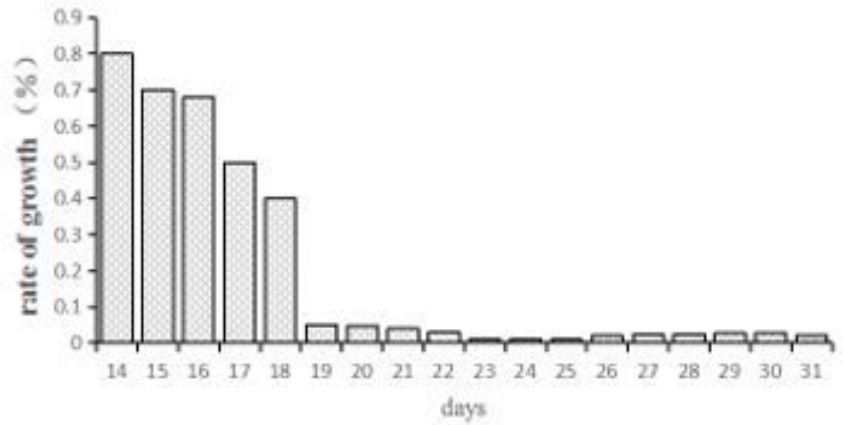

BSMV:00

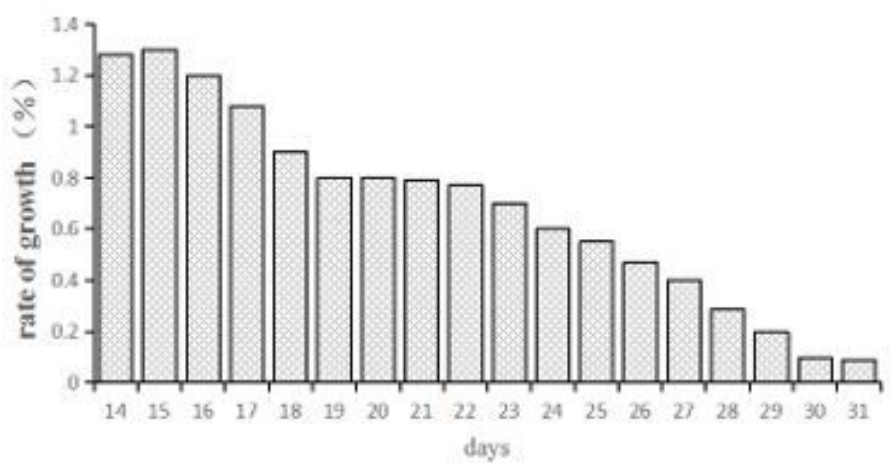

BSMV:Tapcna-2

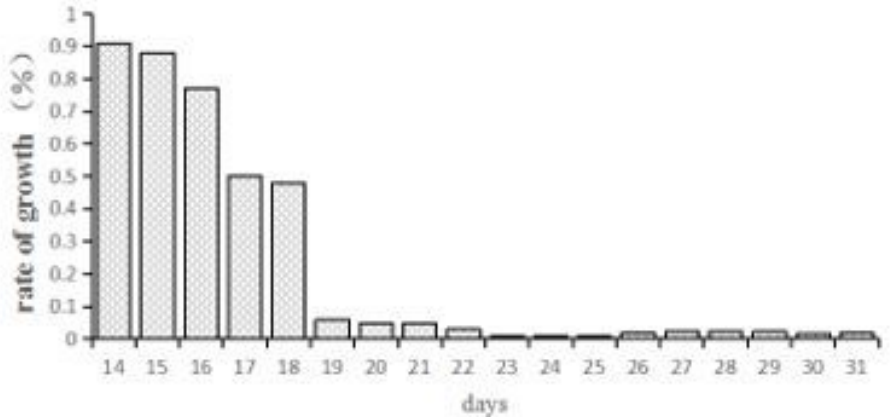

Figure 3

Growth rate of each group.
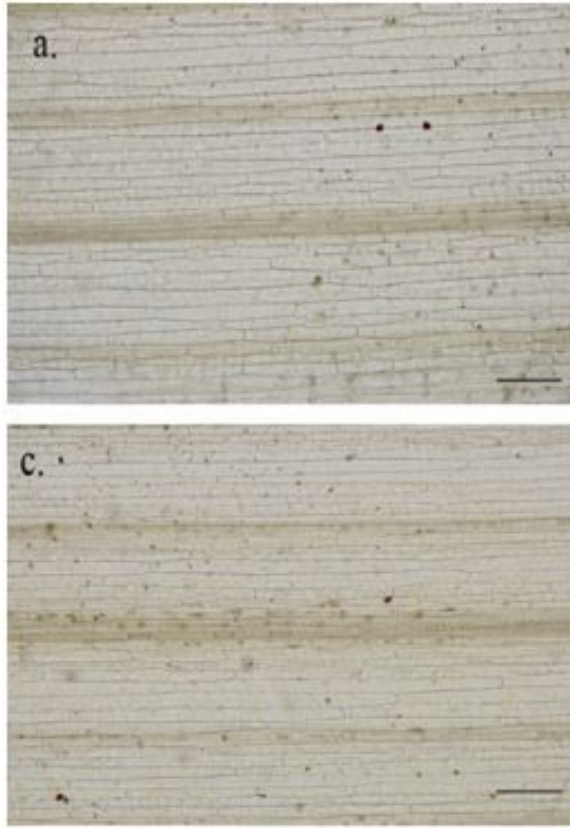
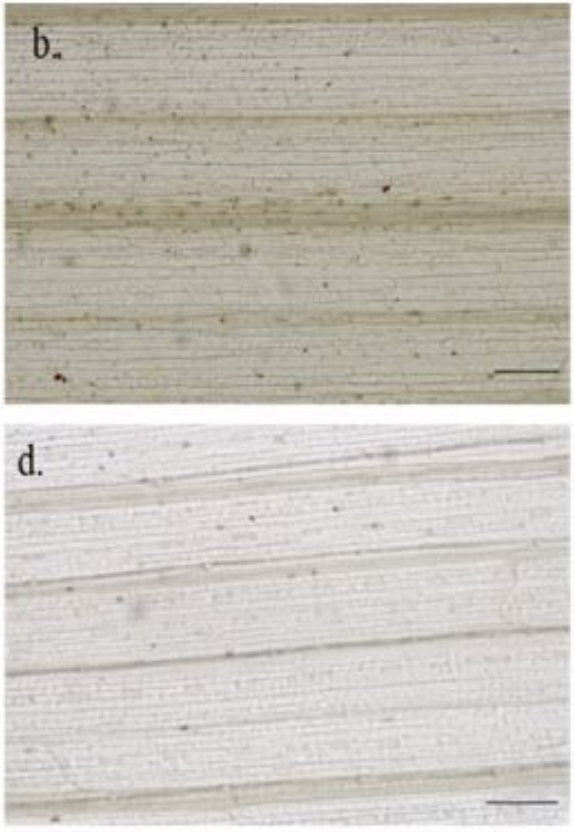

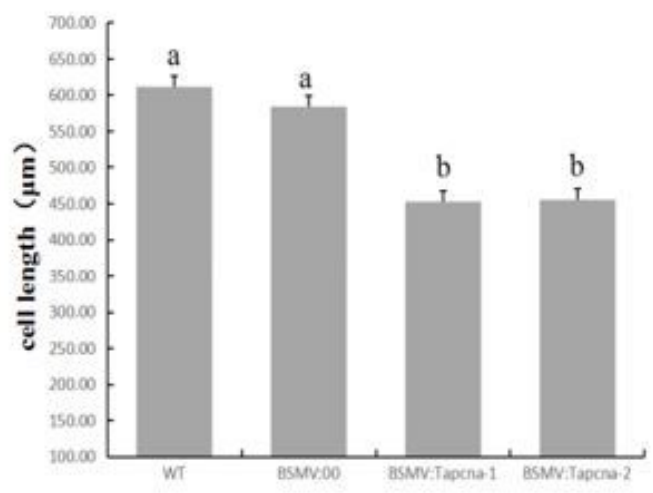


Figure 4

Length of mysophyll cells. a-d Cell morphology of WT, BSMV:00, BSMV:TaPCNA-1 and BSMV:TaPCNA-2 respectively. The histogram shows the cell length.
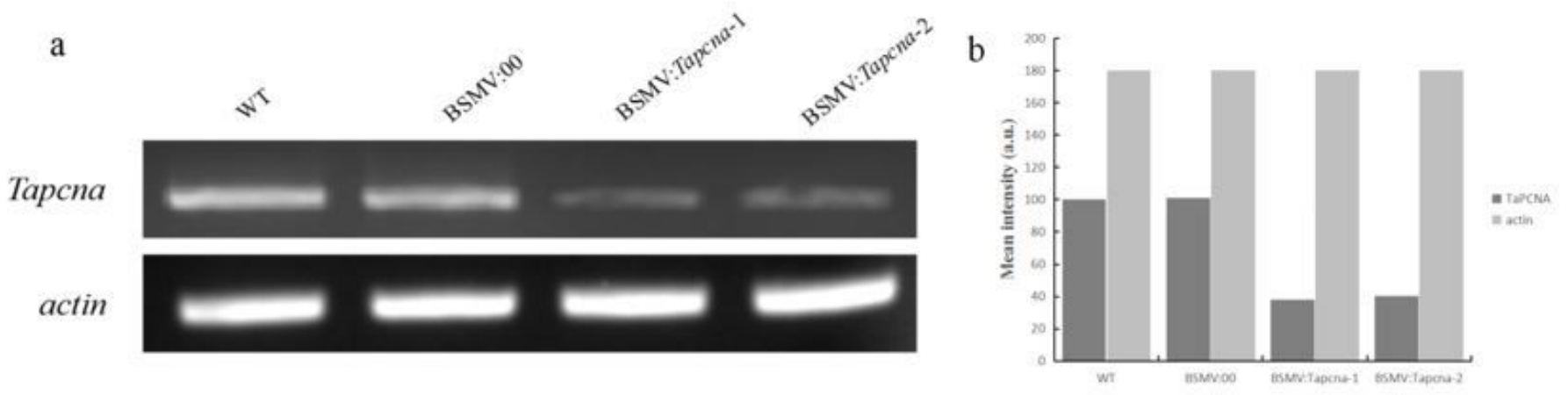

c
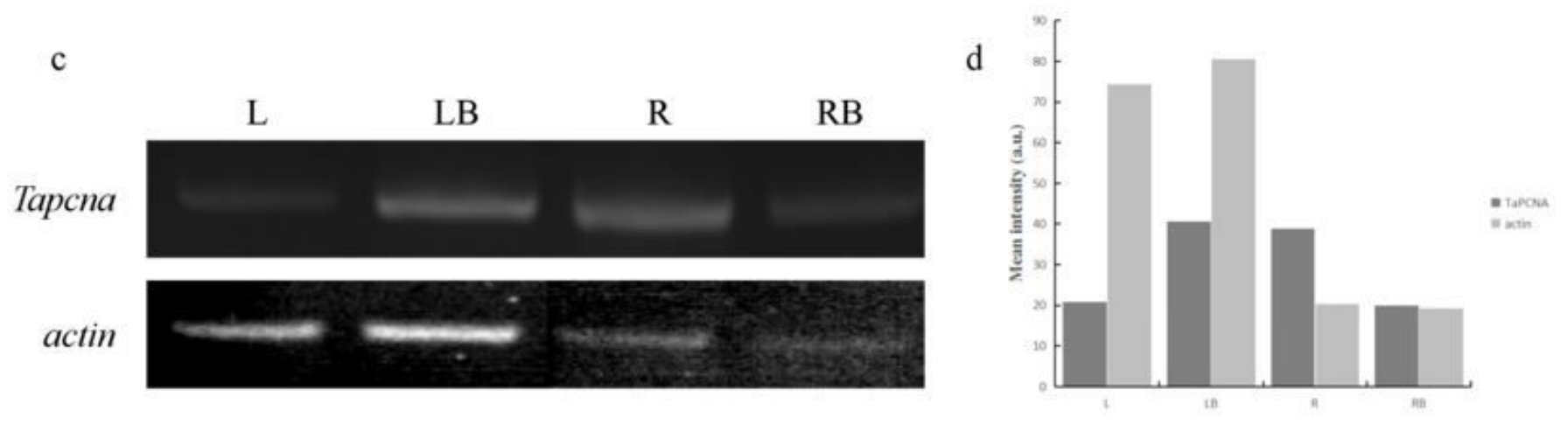

\section{Figure 5}

Semi-quantitative RT-PCR of TaPCNA in different group. (a) detection of gene silencing efficiency. (b) Gray value analysis. (c) L, LB, R, and RB indicate the leaves, leaveas under enhanced UV-B, root and root exposed to UV-B radation. (d) Gray value analysis of the gel. 
a

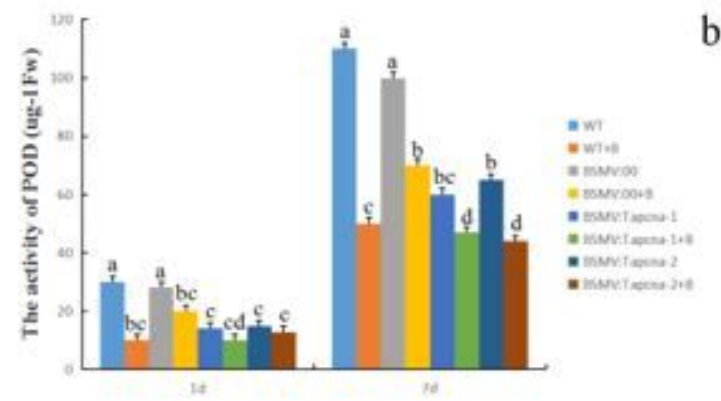

c
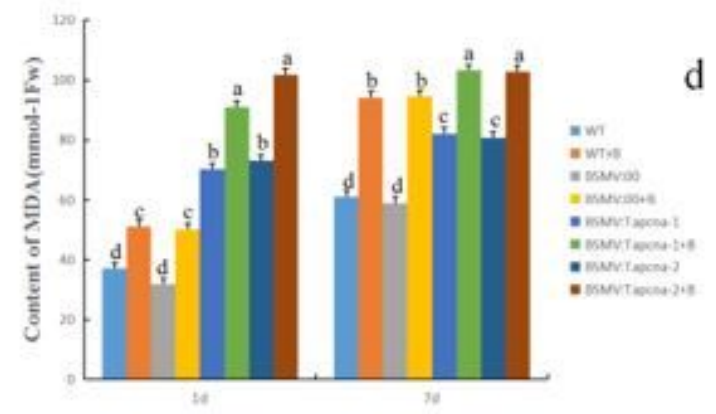

b
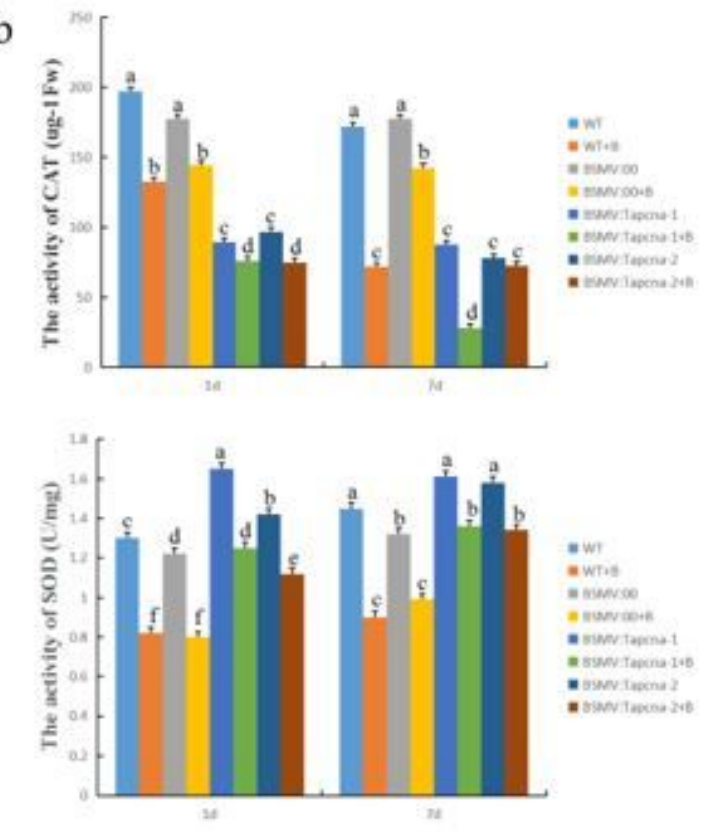

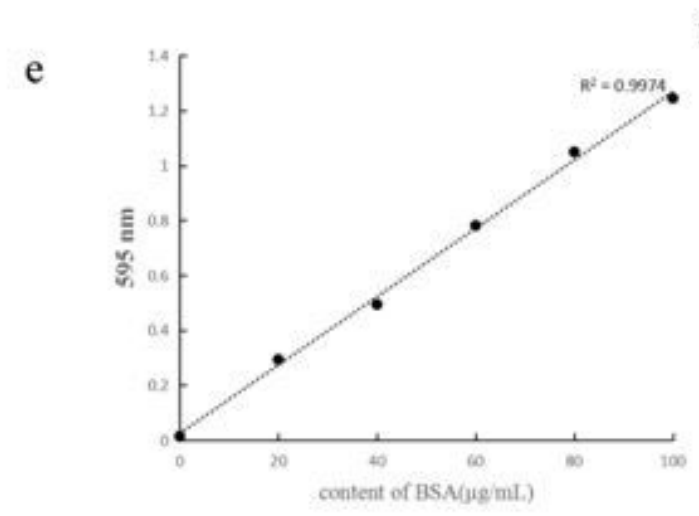

f

$\mathrm{g}$
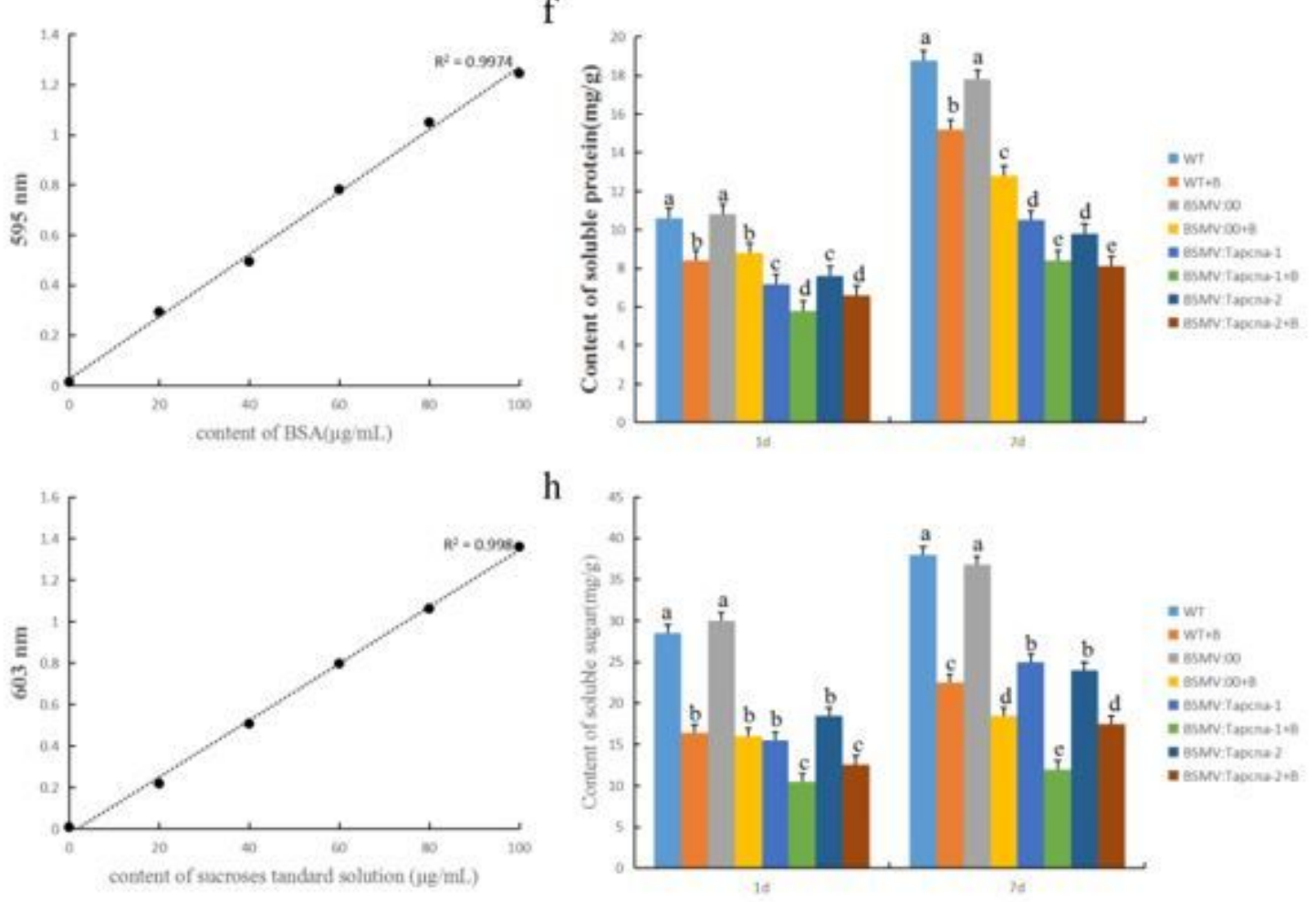

h

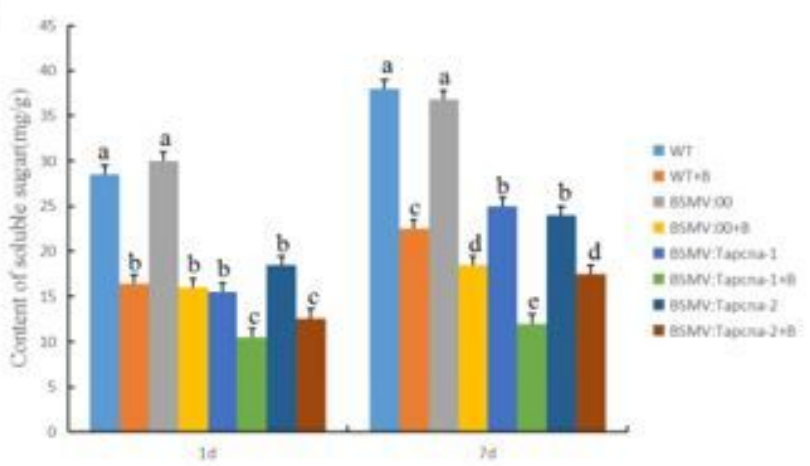

\section{Figure 6}

Determination of physiochemical and metabolisms parameters. (a) The activity of POD in different groups. (b) The activity of CAT in different groups. (c) MDA content in different groups. (d) The activity of SOD in different groups. (e) Standard protein curve. (f) Soluble protein content in different groups. (g) Standard sucrose curve. (h) Soluble sugar content. Data means $\pm S E$, means followed by different letters are significantly different $(P<0.05)$. 


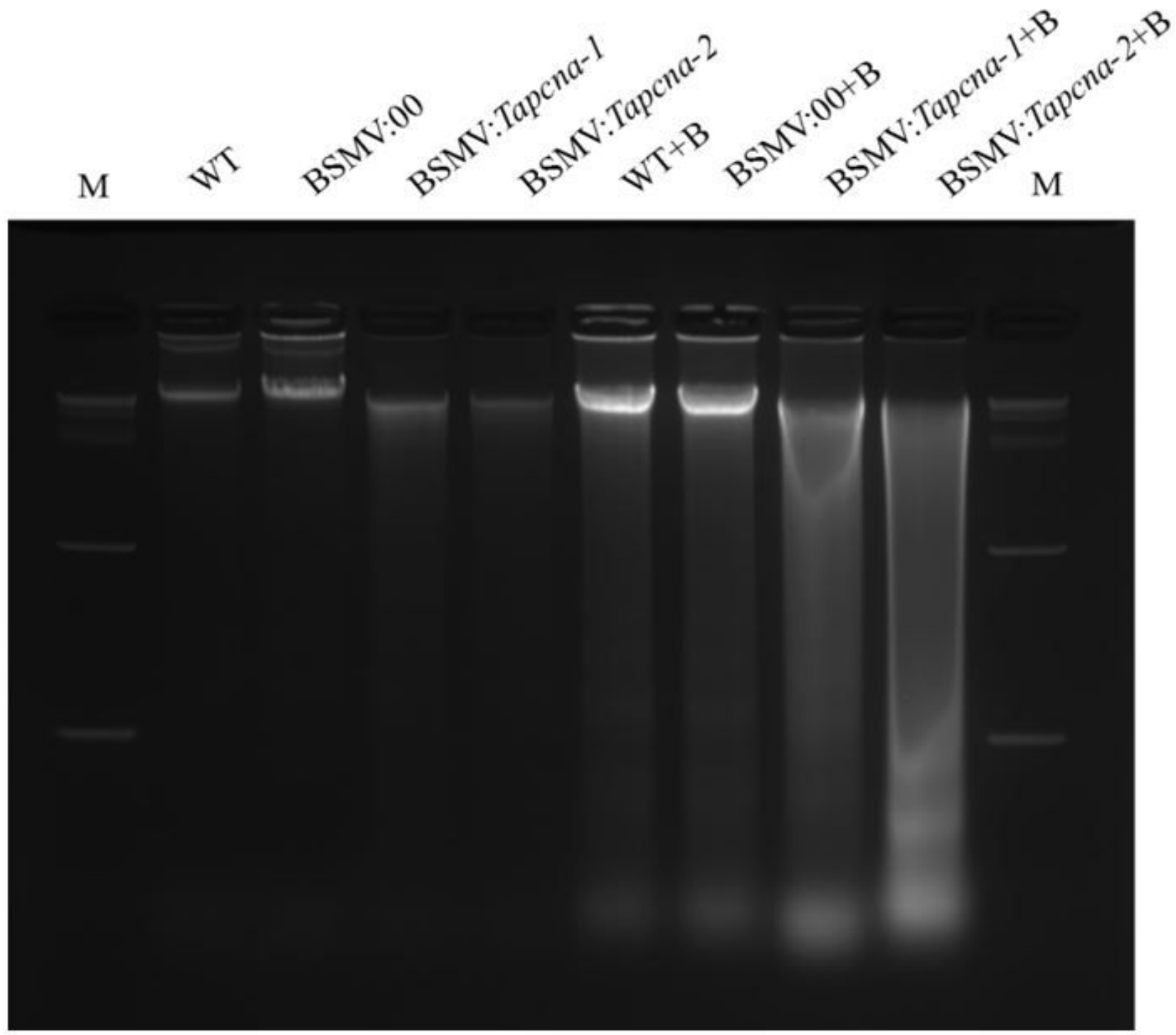

Figure 7

DNA laddering in different treatment. 
A

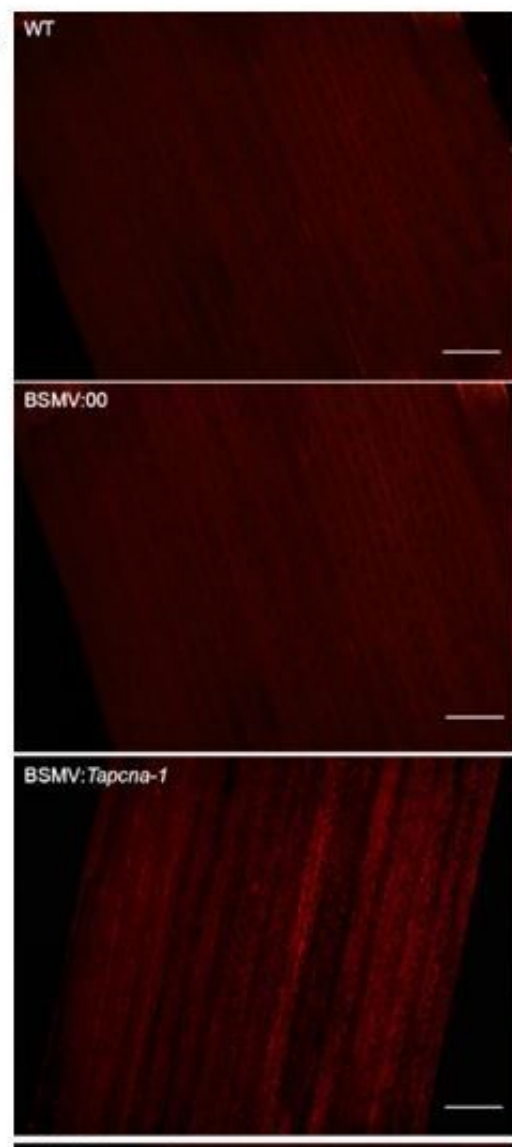

BSMV:00+B

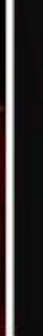

WT+B
B

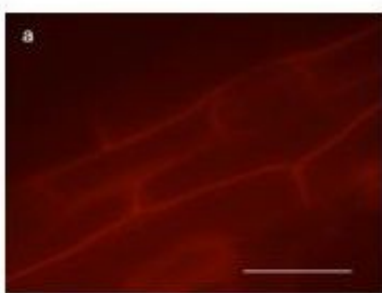

$\circ$

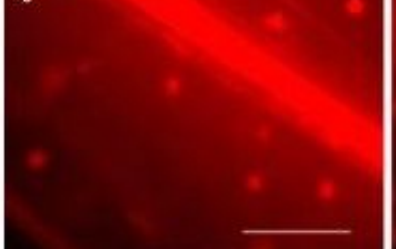

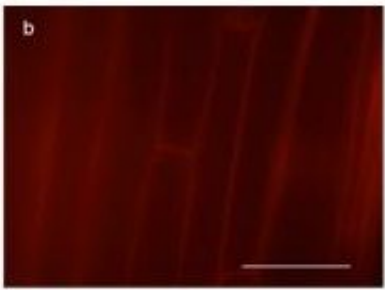

d

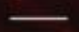

BSMV:Tapcna-1+8

BSMV:Tapcna-2

BSMV:Tapcna-2+8

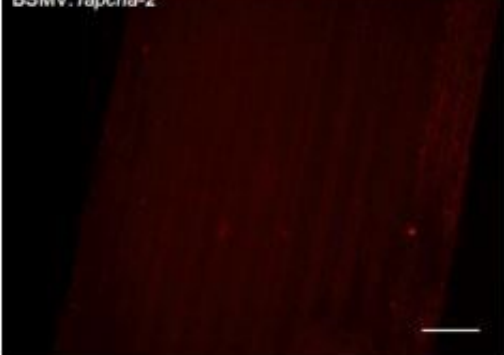

\section{Figure 8}

PI staining in each group. (A) Different treatment groups after PI staining. (B) The a and b stands for normal cells staining, the $\mathrm{c}$ and $\mathrm{d}$ stands for apoptotic nuclear staining, and abnormal chloroplast staining. 